\title{
Tecnura
}

http://revistas.udistrital.edu.co/ojs/index.php/Tecnura/issue/view/640

Estudio DE CASO

\section{Sistema de alta disponibilidad basado en plataforma de virtualización para pequeñas y medianas empresas}

\author{
High availability system based on virtualization platform \\ for small \& medium businesses
}

\author{
Luis Felipe Wanumen Silva*, Darin Jairo Mosquera Palacios**
}

Citation / Para citar este artículo: Wanumen Silva, L. F., \& Mosquera Palacios, D. J. (2014). Sistema de alta disponibilidad basado en plataforma de virtualización para pequeñas y medianas empresas. Revista Tecnura, Edición especial, 231-239.

Fecha de recepción: 4 de febrero de 2014 / Fecha de aceptación: 16 de mayo de 2014

\begin{abstract}
Resumen
La tolerancia a fallos se basa hoy día fundamentalmente en un concepto: redundancia (Guangping, y otros, 2009). La investigación busca proponer una solución para pequeñas y medianas empresas basada en una arquitectura de alta disponibilidad y tolerancia a fallos, soportada en servicios, mediante la duplicación de sus elementos críticos y la disposición redundante de elementos software y hardware que cooperen (Weiping \& Ke, 2006), bien sea en forma activa-activa o activa-pasiva, siempre en forma transparente al usuario final.
\end{abstract}

Palabras clave: confiabilidad, disponibilidad de sistemas, redundancia, tolerancia a fallos, virtualización.

\begin{abstract}
Fault tolerance today is essentially based on a concept: redundancy (Guangping, y otros, 2009). The research aims to propose a solution to small and medium enterprises based on an architecture for high availability and fault tolerance. Services supported based on duplicating its critical elements and placement of software and hardware redundant which cooperate actively-active or active-passive (Weiping \& Ke, 2006), in a way transparent to the end user.
\end{abstract}

Keywords: High availability, fault tolerance, virtualization, SAN / NAS, Pymes, VMware.

\footnotetext{
* Ingeniero de sistemas. Especialista en ingeniería de software, magíster en Ingeniería de Sistemas y Computación. Docente, Universidad Distrital Francisco José de Caldas. Bogotá, Colombia. Contacto:

Iwanumen@udistrital.edu.co

** Ingeniero de sistemas, especialista en teleinformática, magíster en teleinformática; docente, Universidad Distrital Francisco José de Caldas. Bogotá, Colombia. Contacto: dmosquerap@udistrital.edu.co
} 


\section{INTRODUCCIÓN}

Es claro que durante los últimos años el gobierno, la academia y el sector privado, particularmente el financiero, han dirigido sus estrategias de apoyo y promoción de sus servicios hacia la Pyme, al darse cuenta de que es en este sector empresarial donde se puede tener el apoyo para alcanzar un acelerado crecimiento de la economía y aunque siempre se habían considerado importantes, hoy han Ilegado a ser imprescindibles al proyectarse como una de las mejores opciones para lograr la plena reactivación económica, aun con todas sus falencias como es la falta de gestión organizacional, financiera, comercial, administrativa y tecnológica.

Y es en esa última de sus falencias donde, por medio de este proyecto, queremos brindar una solución que mejore las condiciones tecnológicas de una pyme, mediante nuevas plataformas, de modelos (Ge, 2009) y herramientas unas de pago y libres, pero que ajustándolas a las necesidades de las empresas generarían una solución muy eficiente y a bajos costos.

La investigación incentiva el uso de nuevas tecnologías en las pymes mediante la creación de un sistema de alta disponibilidad (Kishor, Ranjith, David, Swami, \& Rick, 2006) basado en plataforma de virtualización, que ofrece a dichas empresas la consolidación de sus recursos tecnológicos (Philippe, 2010) y a su vez garantiza la alta disponibilidad en los servicios (Sung, y otros, 2007) tecnológicos que presta a sus clientes y empleados.

Los servicios y productos implementados en esta plataforma fueron implementaciones CRM, ERP, Gestor Documental y Servidor WEB con licencias libres. La planeación y ejecución del Sistema de Alta disponibilidad se encuentra enmarcada en la metodología de diseño de soluciones empresariales de redes (Terrasson, Briand, Basrour, \& Dupe, 2009) (Koo \& Kwong, 2005).

\section{CARACTERIZACIÓN DE LAS EMPRESAS A LAS QUE VA DIRIGIDO EL SISTEMA}

El sistema de alta disponibilidad va dirigido a empresas colombianas pequeñas y medianas (conocidas por el acrónimo Pyme, lexicalizado como pyme), son empresas con características distintivas, y tiene dimensiones con ciertos límites ocupacionales y financieros prefijados por los Estados o regiones. Las pymes son agentes con lógicas, culturas, intereses y un espíritu emprendedor específicos. Usualmente se ha visto también el término MiPyME (acrónimo de "micro, pequeña y mediana empresa"), que es una expansión del término original, en donde se incluye la microempresa.

La pequeñas y medianas empresas son entidades independientes, con una alta predominancia en el mercado de comercio, quedando prácticamente excluidas del mercado industrial por las grandes inversiones necesarias y por las limitaciones que impone la legislación en cuanto al volumen de negocio y de personal, los cuales si son superados convierten, por ley, a una microempresa en una pequeña empresa, o una mediana empresa se convierte automáticamente en una gran empresa. Por todo ello una pyme nunca podrá superar ciertas ventas anuales o una determinada cantidad de personal.

\section{Importancia de las Pymes}

Las pequeñas y medianas empresas cumplen un importante papel en la economía de todos los países. Los países de la OCDE suelen tener entre $70 \%$ y $90 \%$ de los empleados en este grupo de empresas.2 Las principales razones de su existencia son:

Pueden realizar productos individualizados en contraposición con las grandes empresas que se enfocan más a productos más estandarizados.

Sirven de tejido auxiliar a las grandes empresas. La mayor parte de las grandes empresas se valen de empresas subcontratadas menores para realizar servicios u operaciones que de estar incluidas en 
el tejido de la gran corporación redundaría en un aumento de coste.

\section{Importancia de las Pymes}

La mayor ventaja de una pyme es su capacidad de cambiar rápidamente su estructura productiva en el caso de variar las necesidades de mercado, lo cual es mucho más difícil en una gran empresa, con un importante número de empleados y grandes sumas de capital invertido. Sin embargo, el acceso a mercados tan específicos o a una cartera reducida de clientes aumenta el riesgo de quiebra de estas empresas, por lo que es importante que estas empresas amplíen su mercado o sus clientes.

- Financiación. Las empresas pequeñas tienen mayor dificultad en encontrar financiación a un coste y plazo adecuados debido a su mayor riesgo. Para solucionar esto se recurre a las SGR y capital riesgo.

- Empleo. Son empresas con mucha rigidez laboral y que tienen dificultades para encontrar mano de obra especializada. La formación previa del empleado es fundamental para estas.

- Tecnología. Debido al pequeño volumen de beneficios que presentan estas empresas no pueden dedicar fondos a la investigación, por lo que tienen que asociarse con universidades o con otras empresas.

- Acceso a mercados internacionales. El menor tamaño complica su entrada en otros mercados.
Desde las instituciones públicas se hacen esfuerzos para formar a las empresas en las culturas de otros países.

En Colombia, de acuerdo con la definición de la ley 590 de 2000 y la ley 905 de 2004, la microempresa es toda unidad de explotación económica realizada por persona natural o jurídica, en actividades empresariales, agropecuarias, industriales, comerciales o de servicio, rural o urbana con planta de personal hasta 10 trabajadores y activos de menos de 501 salarios mínimos legales mensuales vigentes. Según la ley 905 de 2004 la pequeña empresa es aquella que cuenta con una planta de personal entre 11 y 50 trabajadores o activos entre 501 y 5000 salarios mínimos legales mensuales vigentes; por último, la mediana empresa es aquella que cuenta con una planta de personal entre 51 y 200 trabajadores o activos totales entre 5001 y 30.000 salarios mínimos legales mensuales vigentes. 6758 .

\section{CARACTERIZACIÓN DE REQUERIMIENTOS QUE DEBE CUMPLIR EL SISTEMA}

El sistema de alta disponibilidad propuesto debe cumplir unos requerimientos mínimos que garanticen su factible implementación en las Pymes. Estos requerimientos se han extractado después de un análisis profundo de necesidades reales de algunas Pymes y se pueden resumir en requerimientos de 1) redundancia, 2) protección y confiabilidad

Tabla 1. Categorización de las empresas.

\begin{tabular}{lcc}
\hline Tipo de empresa & Empleados & $\begin{array}{c}\text { Activos totales (en salario } \\
\text { mínimo legal mensual vigente) }\end{array}$ \\
\hline Microempresa & $1-10$ & Menos de 501 \\
\hline Pequeña empresa & $11-50$ & $501-5000$ \\
\hline Mediana empresa & $51-200$ & $5001-30.000$ \\
\hline Gran empresa & Más de 200 & Mayor que 30.000 \\
\hline
\end{tabular}

Fuente: (República de Colombia, 2000). 


\section{Requerimiento de redundancia}

El sistema debe implementar mecanismos de redundancia (Guangping, y otros, 2009) que garanticen la repetición de datos o hardware de carácter crítico que se quiere asegurar ante los posibles fallos que puedan surgir por el uso continuado.

Las técnicas de redundancia han sido usadas por la industria militar y aeroespacial durante muchos años para alcanzar una alta confiabilidad (Filho, Hashimoto, \& Rosa, 2008). Una base de datos replicada es un ejemplo de sistema distribuido redundante.

Cuando se tienen sistemas críticos que deben estar disponibles y funcionando 24 horas al día, 365 días al año, hay que intentar minimizar los fallos que puedan afectar el funcionamiento normal del sistema. Fallos van a ocurrir, pero existen técnicas y configuraciones que ayudan a tener sistemas redundantes (Terrasson, Briand, Basrour, \& Dupe, 2009), en los que ciertas partes pueden fallar sin que esto afecte el funcionamiento del mismo.

\section{Requerimiento de protección y confiabilidad}

El sistema debe implementar mecanismos de protección y confiabilidad que le permitan realizar los procesos en más de una estación. Estos mecanismos garantizan que si por algún motivo alguno de los sistemas dejara de funcionar o algún servicio colapsara, inmediatamente otro tendría que ocupar su lugar y realizaría las tareas del anterior. (Meng \& Shao-hua, 2005).

\section{Requerimiento de disponibilidad}

El sistema debe implementar mecanismos de alta disponibilidad (Filho, Hashimoto, \& Rosa, 2008), los cuales pueden ir desde bases de datos simplemente replicadas hasta sistemas distribuidos redundantes complejos.

\section{Requerimiento de implementación de cluster}

Las Pymes deben contar con sistemas de tipo cluster (Song, Leangsuksun, Nassar, Gottumukkala, \& Scott, 2006) que les permitan escalar a nivel de datos, de aplicación y de servicios. Aparte de ello, los sistemas de tipo cluster apoyan tecnológicamente la implementación de los mecanismos de redundancia, protección, disponibilidad y confiabilidad.

El cluster se aplica a los conjuntos o conglomerados de computadores construidos mediante la utilización de componentes de hardware comunes y que se comportan como si fuesen un único computador. Los cluster de alta disponibilidad son clusters cuyo objetivo de diseño es proveer disponibilidad y confiabilidad. Estos clusters tratan de brindar la máxima disponibilidad de los servicios que ofrecen (Song, Leangsuksun, Nassar, Gottumukkala, \& Scott, 2006). La confiabilidad se provee mediante software que detecta fallos y permite recuperarse frente a los mismos, mientras que en hardware se evita tener un único punto de fallos.

Los clusters son usualmente empleados para mejorar el rendimiento y la disponibilidad por encima de la que es provista por un solo computador típicamente siendo más económico que computadores individuales de rapidez y disponibilidad comparables.

\section{Requerimientos de replicación}

El sistema para el caso de las Pymes debe soportar mecanismos de seguridad que garanticen el funcionamiento del sistema. Cuantos más componentes existan, mayor probabilidad se tiene de que algo falle. Estos problemas pueden ocurrir en el propio servidor, fallos de discos, fuentes de alimentación, tarjetas de red, etc., y en la infraestructura necesaria para que el servidor se pueda utilizar, componentes de red, acceso a internet, sistema, etc. Surge entonces el concepto de replicación que se puede utilizar para replicar datos en un servidor en espera, lo que proporciona mayor disponibilidad en caso de inactividad prevista 
o imprevista del sistema. Para el caso de las Pymes, los mecanismos de replicación se utilizan para proporcionar datos en espera semiactiva si los datos requeridos en el servidor en espera son un subconjunto de los datos necesarios en el servidor principal (Zhengping, Juyang, \& Prokhorov, 2008).

\section{SELECCIÓN DE LA ALTERNATIVA TECNOLÓGICA}

Atendiendo a los requerimientos anteriores, PNP Manager utiliza un cluster que responde a las siguientes necesidades:

1. Alto rendimiento

2.Alta disponibilidad (Filho, Hashimoto, \& Rosa, 2008)
3. Balanceo de carga
4. Escalabilidad

La disponibilidad de infraestructuras como las citadas anteriormente ofrecen diversos escenarios en donde pueden desplegarse aplicaciones distribuidas. Esto garantiza que implementando una solución basada en PNP Manager, se pueden implementar los requerimientos identificados en la sección anterior. Teniendo en cuenta que un requerimiento de implementar un cluster afecta otros requerimientos como el de disponibilidad, se tiene una relación entre requerimientos. Se podría pensar entonces que al implementar PNP Manager con VMWare se logra satisfacer los requerimientos de la sección anterior. Una vez se ha seleccionado la tecnología a implementar en las Pymes se usa la metodología Top Down Network Desing (Koo \& Kwong, 2005) para implementar esta solución en dichas empresas. No obstante, en la implantación de esta metodología, nuevamente se hacen revisiones de las necesidades tecnológicas de las Pymes. Esto no es un error, sino que da la posibilidad de crear un diseño más detallado de sistema de alta disponibilidad que cumpla con las expectativas de las Pymes.

\section{METODOLOGÍA}

La implementación del sistema de alta disponibilidad basado en plataforma de virtualización para pequeñas y medianas empresas se realiza utilizando la metodología Top Down Network Desing (Koo \& Kwong, 2005).

Top Down es una metodología que guía el diseño de soluciones empresariales de redes confiables, seguras y administrables; presenta la estructura que se muestra en la figura 1.

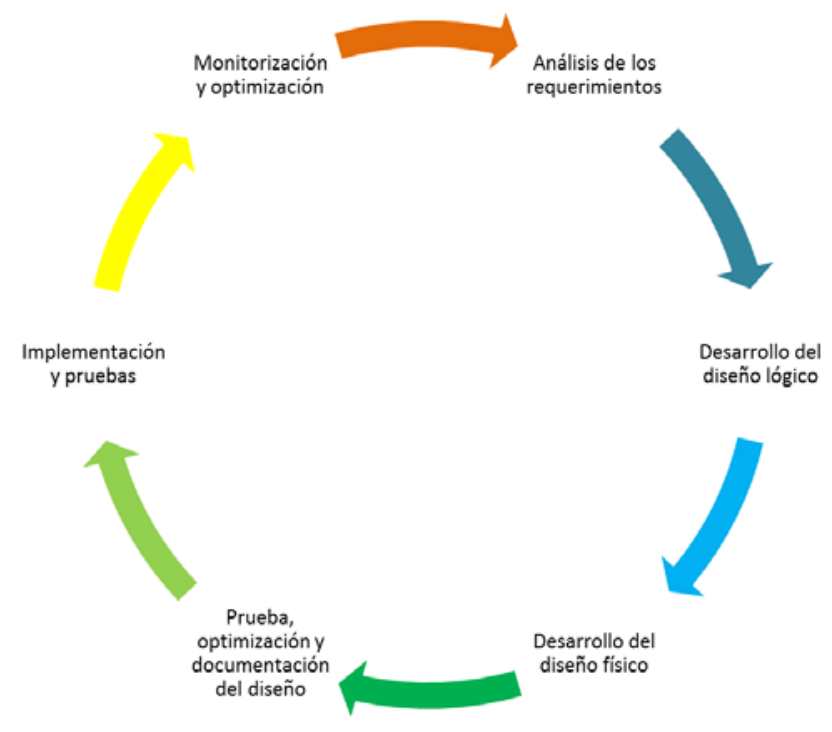

Figura 1. Etapas de la metodología Top Down (Koo \& Kwong, 2005). 
Las fases del enfoque Top-Down cubre (Koo \& Kwong, 2005): 1) Análisis de los requerimientos: Qué debe hacer el sistema. Se refiere básicamente a una necesidad documentada sobre el contenido, forma o funcionalidad de un producto o servicio. 2) Desarrollo del diseño lógico: El diseño de un sistema responde a la forma en que el sistema cumplirá con los requerimientos identificados durante la fase de análisis. 3) Desarrollo del diseño físico: Hace referencia al desarrollo e instalación de software. ¿Cómo se va a hacer? Estudio de alternativas y diseño arquitectónico. 4) Prueba, optimización y documentación de diseño: Consiste en la elaboración de la "descripción formal" del sistema: manuales de usuario, manuales del sistema y manuales de procedimiento. 5) Implementación y pruebas: adquisición, creación e integración de los recursos necesarios para que el sistema funcione. 6) Monitorización y optimización: Consiste en la elaboración de cambios pertinentes para que el sistema se ejecute y funcione más rápidamente.

\section{DESCRIPCIÓN DE LA SOLUCIÓN}

Atendiendo a los objetivos de las pymes en la actualidad se requiere una infraestructura tecnológica que garantice una operación continua sobre los servicios que ofrece las pymes tanto a sus empleados como a los clientes obteniendo una mayor competitividad, eficiencia y escalabilidad que le permitan lograr un reconocimiento dentro del campo productivo como generador de soluciones innovadoras haciendo uso de las tecnologías de información (TI).

Para esto, se propone la implementación de una solución telemática en la que se desarrolle una infraestructura tecnológica que garantice el adecuado funcionamiento y dé respuesta a las solicitudes de los usuarios de la aplicación web "PNP Manager".

PNP Manager es una aplicación web que permite obtener un modelo de usuario que define las preferencias de accesibilidad a recursos digitales basado en el estándar ISO/IEC 24751 que integra tres niveles: presentación, control y contenido.
Para garantizar el alto rendimiento de PNP Manager se requiere implementar una solución telemática que asegure tolerancia a fallos, transparencia, escalabilidad y compartición de recursos, con el fin de lograr una mejor experiencia de usuario en el uso de la aplicación.

Para garantizar alta disponibilidad y rendimiento de PNP Manager se implementa un sistema distribuido compuesto por un servidor Web Apache, dos servidores de aplicación JBoss y dos servidores de bases de datos Postgresql.

Sin embargo, para la implementación de la solución es necesario considerar las siguientes restricciones: 1) La solución se debe implementar utilizando software libre debido a que no se cuenta con recursos económicos para hacer uso de tecnologías propietarias. 2) La solución se debe implementar haciendo uso de los equipos disponibles en el grupo de investigación Metis.

Atendiendo a los requerimientos y a las restricciones establecidas, se implementa un cluster de balanceo de cargas que cumple los siguientes objetivos: mejorar el rendimiento de la aplicación, asegurar tolerancia a fallos, ser transparente, ser escalable y facilitar la compartición de recursos. Una alternativa de solución es la implementación de una solución de virtualización basada en VMWare (Philippe, 2010), la cual permite configurar sistemas operativos alternos a un sistema operativo principal que permita al sistema responder en caso de que el principal falle, tal como lo muestra la arquitectura de VMWare de la figura 2.

En un cluster de balanceo de cargas se debe identificar la forma en que las peticiones se distribuirán a través de varios servidores. Para ello, se recomienda una arquitectura de cluster de balanceo de cargas similar a la presentada en la figura 3, en donde se observa que las peticiones hechas por un browser son recibidas en primera instancia por un equipo "Balanceador de cargas" que tiene como función determinar basado en unas políticas, cuál de los nodos del cluster está disponible en ese instante para resolver las peticiones del cliente. 


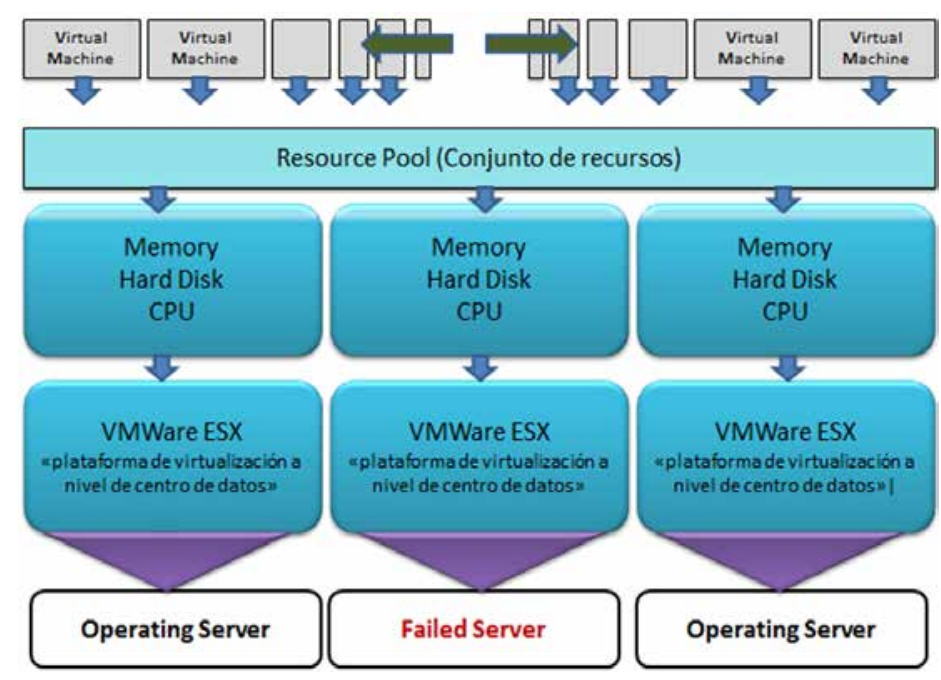

Figura 2. Arquitectura de Alta Disponibilidad VMware (Philippe, 2010).

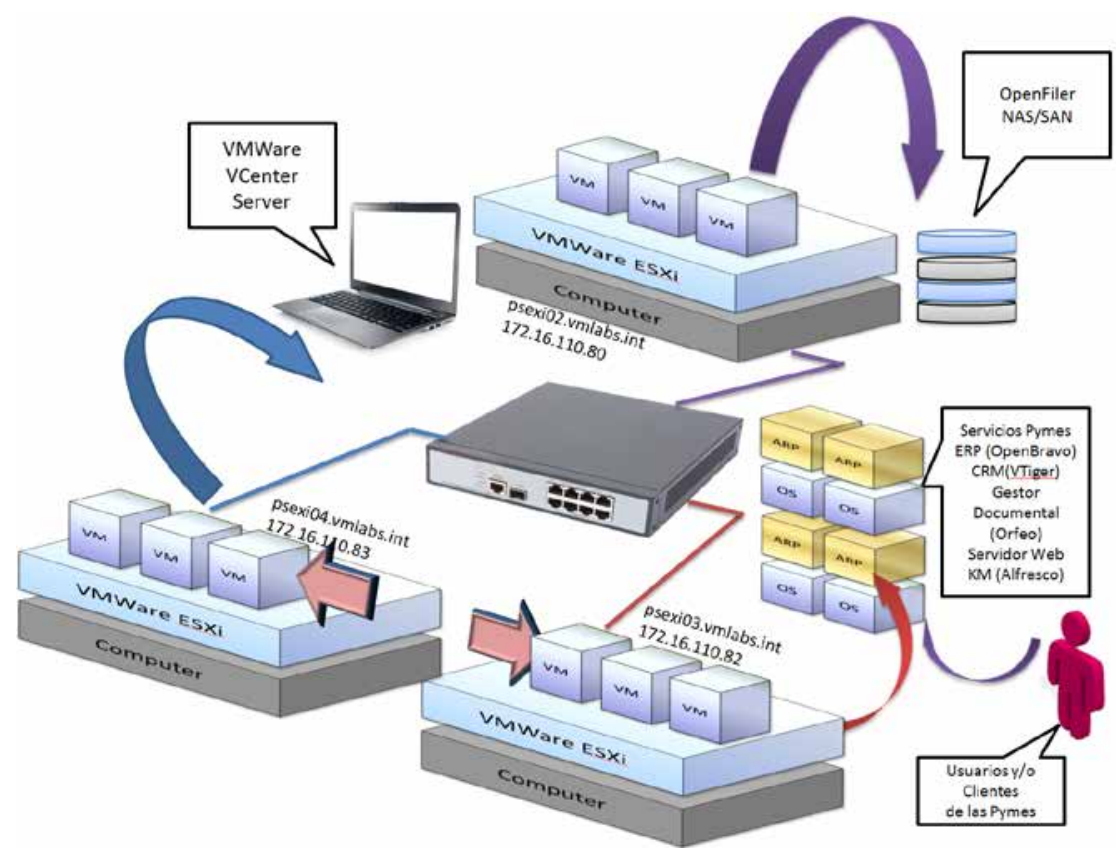

Figura 3. Arquitectura para sistemas de alta disponibilidad basada en VMWare (Philippe, 2010).

Para implementar el sistema de alta disponibilidad propuesto se requiere inicialmente tres servidores y con el propósito de garantizar la escalabilidad, se concluye que el actual esquema de direccionamiento utilizado permite la asignación de los nombres y direcciones mostrados en la tabla 2.
Tabla 2. Asignación de nombres y direcciones a los equipos del sistema de Alta Disponibilidad.

\begin{tabular}{lc}
\hline Nombre & IP \\
\hline psesxi02.vmlabs.int & 172.16 .110 .80 \\
\hline psesxi03.vmlabs.int & 172.16 .110 .82 \\
\hline psesxi04.vmlabs.int & 172.16 .110 .83 \\
\hline
\end{tabular}

Fuente: Elaboración de los autores. 
En la implementación de este prototipo no se utiliza servidor de dominio, por lo que se utilizará el archivo hosts de cada servidor para facilitar la resolución de nombres y direcciones IP.

Una parte importante de todo el sistema de alta disponibilidad es el almacenamiento compartido, para lo cual se utilizó un Appliance de SAN Ilamado Openfiler el cual presenta a través de iSCSI las unidades lógicas (LUNS) a cada uno de los HOSTS (ESXi); de esta manera las máquinas virtuales en que se encuentra cada uno de los servicios ofrecidos por las pymes están almacenadas en la SAN y no en los discos locales, lo cual permitirá que en el momento de una caída por parte de los HOSTS, el otro pueda tomar posesión de las máquinas y prenderlas de manera automática.

\section{RESULTADOS}

La solución telemática propuesta asegura alta disponibilidad, consolidación, escalabilidad y compartición de recursos, garantizando que los servicios que ofrecen las pymes se mantengan disponibles de manera continua.

La plataforma de virtualización en la solución de alta disponibilidad brinda muchos beneficios, ya que la administración de los servicios es muy fácil y el ahorro en infraestructura se hace evidente.

Se demuestra que VMware en el mercado de virtualización es el gran líder y que sus productos ofrecen mejores beneficios que el resto de la competencia, y que aun siendo de pago tiene precios razonables para que una pyme tenga oportunidad de adquirirlos.

Se evidencia el gran potencial de herramientas OpenSource ya que en gran parte de toda la solución nos apoyamos en ellas y se observa la gran madurez de desarrollo en que se encuentran.

Un aspecto muy interesante a nivel de seguridad es que el sistema operativo ESXi es muy liviano y robusto, lo que le permite tener un alto desempeño y a su vez muy seguro; adicionalmente los demás sistemas operativos para esta solución están basados en Linux, lo que garantiza la posibilidad de asegurarlo bajo las mejores prácticas.

\section{CONCLUSIONES}

El estado del arte realizado para determinar las soluciones existentes sugiere que el proyecto es una idea innovadora y que su proyección social permitirá asistir a todos aquellas empresas pequeñas o medianas que deseen acceder a tecnología de gran escala a costos razonables.

La utilización de la metodología Top Down facilitó el desarrollo e implementación de este proyecto. Top Down guió la implementación del sistema de alta disponibilidad.

Con la implementación del sistema de alta disponibilidad se evidenciaron las grandes ventajas que tiene la plataforma de virtualización, y cómo esta puede apoyar soluciones de consolidación y alta disponibilidad en la infraestructura de las pymes.

El sistema de alta disponibilidad mostró el aprovechamiento de los recursos de infraestructura y generó un modelo de infraestructura que puede ser escalable en la medida de los recurso de las pymes.

Las pruebas fueron exitosas y evidenciaron paso a paso lo que sucedía con el sistema de alta disponibilidad y cómo realiza el failover, ofreciendo continuidad en los servicios en tiempos óptimos.

\section{FINANCIAMIENTO:}

Universidad Distrital Francisco José de Caldas

\section{REFERENCIAS}

Filho, E.; Hashimoto, G., \& Rosa, P. (2008). A High Availability Firewall Model Based on SCTP Protocol. ICSNC '08. 3rd International Conference on (pp. 2002-2007). Systems and Networks Communications.

Ge, G.-f. (2009). Research on the Implementation Method of Document Management in PDM System. 2009 International Conference on Signal Processing Systems (pp. 707-710). Singapore: ICSPS. 
Guangping, X.; Yong, M.; Wenhui, M.; Gang, W.; Xiaoguang, L., \& Jing, L. (2009, May). Model and Evaluation of Redundant Data Organization for High Availability in Structured Overlay Networks. Software Engineering, Artificial Intelligences, Networking and Parallel/Distributed Computing, 447-452.

Kishor, T. S.; Ranjith, V.; David, T.; Swami, N., \& Rick, C. (2006). Modeling High Availability. PRDC '06. 12th Pacific Rim International Symposium on (pp. 154164). Dependable Computing.

Koo, S., \& Kwong, S. W. (2005). Teaching Computer Communication Networks: Top-down or Bottom-up? FIE '05. Proceedings 35th Annual Conference (pp. 19-22). Frontiers in Education.

Meng, J., \& Shao-hua, Y. (2005). Modeling and analysis of high-availability routing software. Wireless Communications, Networking and Mobile Computing, 2005 (pp. 1045-1048). 2005 International Conference on.

Philippe, G. (2010). Virtualización de sistemas de información con VMWare. Arquitectura, proyecto, seguridad y feedbacks. Ediciones EDI. Informática Técnica.

República de Colombia (2000, mayo 15). Ley 590 de 2000. Periódico de la Secretaría del Senado, pp. 12-13.
Song, H.; Leangsuksun, C.; Nassar, R.; Gottumukkala, N. R., \& Scott, S. (2006). Availability modeling and analysis on high performance cluster computing systems. The First International Conference (pp. 2022). Availability, Reliability and Security.

Sung, H.; Choi, B.; Kim, H.; Song, J.; Han, S.; Ang, C.W., . . . Wong, K.-S. (2007). Dynamic Clustering Model for High Service Availability. ISADS '07. Eighth International Symposium on (pp. 311-317). Autonomous Decentralized Systems.

Terrasson, G.; Briand, R.; Basrour, S., \& Dupe, V. (2009). A top-down approach for the design of low-power microsensor nodes for wireless sensor network. FDL 2009. Forum on (pp. 1-6). Specification \& Design Languages, 2009.

Weiping, Z., \& Ke, D. (2006). A Top Down Approach to Estimate Network Loss Rate. Communications and Networking in China, 2006. ChinaCom '06. First International Conference on, (pp. 1-5). China.

Zhengping, J.; Juyang, W., \& Prokhorov, D. (2008). Where-what network 1: "Where" and "what" assist each other through top-down connections. Development and Learning (pp. 61-66). ICDL 2008. 7th IEEE International Conference on.

\section{(9) $(1) \Theta \Theta$}


